\title{
El gobierno de Evo Morales y El FANTASMA DEL NACIONALISMO REVOLUCIONARIO DEL 52 EN BOLIVIA. HACIA UNA “INDIANIZACIÓN" Y PLURALIZACIÓN DEL CONCEPTO DE POdER dUAl de RenÉ Zavaleta MERCAdo
}

\author{
Evo Morales Government and the Spectre of \\ ReVOlutionary NATIONALISM OF 52 IN Bolivia. \\ Towards an “Indianization" and Pluralization of the \\ Concept of Dual Power of René Zavaleta Mercado
}

\author{
Mamoru Fujita* \\ doi: https://doi.org/10.31644/ED.V9.N1.2022.A15
}

Resumen: Este artículo analiza el proceso de la política boliviana después de la llegada de Evo Morales Ayma a la presidencia (2005) en su relación con el gobierno de Carlos Mesa Gisbert (2003-2005). En base a un intento de ampliar e "indianizar" el concepto de poder dual del pensador político boliviano René Zavaleta Mercado, se argumenta que el gobierno de Morales, en vez de construir una agenda y un poder verdaderamente plurinacional basados en la "Agenda de octubre" de 2003, se acercó cada vez más al nacionalismo revolucionario de 1952, lo que últimamente hizo vulnerable a Morales y el gobierno de MAS (Movimiento al Socialismo) ante Mesa en las elecciones generales de 2019.

Palabras clave: indianismo, René Zavaleta Mercado, poder dual, nacionalismo revolucionario, plurinacionalismo.

Summary: This article analizes the political process of Bolivia since the onset of the Evo Morales Ayma government (2005) in its relationship with the government led by Carlos Mesa Gisbert (2003-2005). Based on an attempt to broaden, from the indianista point of view, the concept of dual power elaborated by the Bolivian political theorist René Zavaleta Mercado, it is argued that

\footnotetext{
* Mtro. en Estudios del Desarrollo, colaborador externo del Taller de Historia Oral Andina, La Paz, Bolivia; profesor asistente, Facultad de Estudios de Medio Ambiente e Información, Universidad de Keio en Shonan-Fujisawa, Fujisawa, Japón. Correo-e: mfujita1023@gmail.com; mfujita@sfc.keio.ac.jp.

Fecha de recepción: 17/05/2021. Fecha de aceptación: 02/12/2021. Fecha de publicación: 31/01/2022.

(cc) BY-NC-ND

EntreDiversidades. Revista de Ciencias Sociales y Humanidades, Vol. 9, Núm. 1 (18), enero-junio 2022. Páginas: $360-381$ ISSN-e: 2007-7610. https://doi.org/10.31644/ED.V9.N1.2022.A15 
the Morales government, instead of building a truly plurinational agenda and power based on the "October Agenda" of 2003, drew nearer to the revolutionary nationalism of 1952, which ultimately made Morales and the MAS government vulnerable to Mesa in the general elections of 2019.

Keywords: indianismo, René Zavaleta Mercado, dual power, revolutionary nationalism, plurinationalism.

\section{Introducción: El gobierno de Evo Morales y nacionalismo}

La victoria de Evo Morales en las elecciones generales de diciembre de 2005 fue percibida por el analista James Dunkerley (2007) como la "tercera revolución boliviana”, después de la independencia de 1825 y la revolución "nacional" de 1952. El proceso de movilizaciones sociales a partir del año 2000 que culminó en dicha victoria electoral —considerada como la "refundación" del Estado que definió a Bolivia como un "Estado Plurinacional" a través de la nueva Constitución de 2009—, dieron una fuerte impresión del ascenso indígena al poder y de una nueva forma de hacer política (Fujita, 2009).

Sin embargo, en la siguiente década, la de 2010, la sensación de un "giro a la derecha" del gobierno se fue manifestando cada vez más (Rivera Cusicanqui, 2015). A pesar de la incorporación de los conceptos "indígenas" de suma qamaña (buen vivir) en el Plan Nacional de Desarrollo, el gobierno de Morales volvió al estilo tradicional de buscar el desarrollo mediante la inversión en infraestructura, como en el caso de la integración vial. Simbolizado por el conflicto del TIPNIS (Territorio Indígena Parque Nacional "Isiboro Securé") —en el cual los pueblos originarios del oriente, con la solidaridad de algunas organizaciones indígenas del occidente y de la clase media urbana, protestaron en contra de la intención del gobierno central de construir una carretera que atraviesa su territorio - el gobierno se mostró tendente a priorizar su proyecto en perjuicio de la vivencia de los pueblos originarios. A su vez, manifestó cada vez menos disposición a dialogar y negociar con las movilizaciones de protesta y más bien intentó ganarles por la fuerza. El consiguiente malestar generalizado de la población boliviana estuvo detrás de la caída del apoyo a Evo Morales y su partido Movimiento al Socialismo (MAS) en las elecciones generales de 2019 y el consecuente desborde de protestas. Además, todo ello aumentó la intención de la derecha de desestabilizar la situación.

¿Cómo explicar este aparente cambio en la postura del gobierno de Evo Morales? La interpretación dominante ha sido el enfoque sobre el extractivismo (Marston y Kennemore, 2019; Wanderley, 2018; Lalander, 2017, entre otros). En respuesta a la creciente demanda de materia prima en el mercado global, la región latinoamericana ha vuelto a su viejo patrón de exportación para suscitar crecimiento económico, generando una tendencia que se denomina como "el consenso de materias primas" (commodities consensus) (Svampa, 2013, entre otros), lo que dificulta al gobierno de cada país experimentar con políticas alternativas. Bolivia, un país donde su modelo político depende de los ingresos generados por el sector de hidrocarburos, no es una excepción a esta tendencia general. Sin embargo, igual que sucede con las teorías de la

EntreDiversidades. Revista de Ciencias Sociales y Humanidades, Vol. 9, Núm. 1 (18), enero-junio 2022. Páginas: $360-381$ ISSN-e: 2007-7610. https://doi.org/10.31644/ED.V9.N1.2022.A15 
dependencia, una explicación económica que no toma en cuenta las variadas respuestas políticas que pueden influir al curso económico, queda incompleta (Stern, 1988). Además, el rechazo inicial por parte del gobierno boliviano a dialogar con los movimientos indígenas iría en contra del estándar global de consulta previa. Indudablemente, se puede detectar aquí un factor político en juego. ${ }^{1}$

Debemos recordar que desde la etapa inicial del gobierno de Evo Morales se detectaba un conflicto en su línea política entre la nueva ideología plurinacionalista y el "viejo" nacionalismo originado en la revolución del 1952, basado en la idea del mestizaje (Mayorga y Rodríguez, 2013). Anterior a esto, Antezana (2006) había advertido que el nacionalismo revolucionario no había perdido su relevancia a pesar de las movilizaciones indígenas y las posturas indianistas que estos asumían, o del aparente desinterés del gobierno y del público en general en conmemorar el abril de 1952. Dunkerley (2013) concuerda con esta visión, y ve una semejanza con el período de gobierno del MAS, el cual considera como "otra revolución traicionada, conducida pasivamente" (2013: 327). A estos trabajos se suma la crítica aymara-indianista, a través de varios números de la revista Willka, sobre los "entornos blancoides" de su gobierno, según la cual los cargos importantes en el gobierno de Morales estaban ocupados por los sectores blanco-mestizos de la sociedad boliviana, a pesar la proclama de Morales de tener un gobierno de indígenas y de movimientos sociales (Autores varios, 2007). Gutiérrez (2011) argumentó que la prematura llegada al poder de Evo Morales y su partido MAS había generado una situación en la que asumieron el poder sin todavía tener una base ideológica suficientemente amplia. Lo que señala Gutiérrez nos hace recordar la importancia que René Zavaleta Mercado (1937-1984), el pensador político más influyente de Bolivia del siglo XX, dio al concepto de "conciencia".

Este artículo $^{2}$ retoma estas vertientes de reflexiones políticas, y desarrolla la hipótesis de que la compleja relación de Evo Morales con Carlos Mesa Gisbert, quien dirigió el gobierno entre 2003 y 2005, ha seguido repitiéndose en los años posteriores, y que el nacionalismo revolucionario simbolizado en la figura de Carlos Mesa — y también Eduardo Rodríguez Veltzé, quien lo sucedió

\footnotetext{
${ }^{1}$ Cabe mencionar aquí también que hay una vertiente importante de investigaciones que considera al gobierno de Evo Morales y al MAS como un gobierno indígena y que caracteriza su período de administración como nacionalismo indígena, pero con limitaciones para cuestionar la tendencia neoliberal y solucionar las tareas de reparación de las violaciones de los derechos humanos del pasado (Goodale, 2019; Postero, 2017). Igual que las obras mencionadas arriba, admitimos aquí su valor y contribución, así como también los cambios fundamentales que el gobierno de Morales trajo para Bolivia. Sin embargo, este artículo mantiene que es necesario continuar preguntando desde una perspectiva más radical qué significa ser indígena y qué significa un Estado verdaderamente indígena, y relacionarlo con la cuestión del poder y la construcción de un poder indígena, basándonos en las importantes vertientes de pensamiento político boliviano.

${ }^{2}$ La contribución de este artículo y de otros al presente número de la revista EntreDiversidades nace de un ámbito académico y fraternal del denominado "Grupo de estudios FOKUS" conformado en 2013 por Mamoru Fujita, Tadashi Obara, Ei Kawakami, Kahori Umezaki y Toru Shimizu, investigadores sobre todo acerca de temas indígenas de Abya-Yala/las Américas, en el cual nos reunimos mensualmente para avanzar con nuestra investigación. En los ańos 2019 y 2020 tuvimos dos oportunidades de reunirnos con Gracia Imberton, José Luis Escalona y Fumiko Sukikara, primero en San Cristóbal de Las Casas, México, y después en Tokio, Japón. Agradecemos esta oportunidad para fortalecer los lazos e intercambios académicos entre las dos partes, al igual que agradecemos a los dictaminadores por su intercambio crítico y fructífero.
}

EntreDiversidades. Revista de Ciencias Sociales y Humanidades, Vol. 9, Núm. 1 (18), enero-junio 2022. Páginas: 360-381 ISSN-e: 2007-7610. https://doi.org/10.31644/ED.V9.N1.2022.A15 
en el gobierno de transición durante el año 2005 - funciona como un fantasma que cada vez más reviene en la política boliviana, tentando a Evo Morales y su gobierno a acercarse a esa posición política y finalmente a adoptarla. Ser tentado por esta corriente implica que difícilmente podría escaparse del ámbito de influencia de Carlos Mesa, lo que explica el sorprendente número de votos que adquirió este candidato y su asociación ciudadana en los comicios generales de 2019, y también (aunque en menor grado) en los de 2020. Así, en el primer apartado de este artículo, recurrimos a las reflexiones teóricas que desarrolló René Zavaleta Mercado sobre el "poder dual", y argumentamos la necesidad de ampliar el concepto más allá del marxismo y de reinterpretarlo desde una perspectiva indianista, es decir, más allá de "indianizarlo", e intentaremos explicar y analizar el proceso político boliviano de las últimas dos décadas bajo este enfoque. ${ }^{3}$

En la siguiente sección, abordaremos primero el concepto y la temática del poder dual desarrollado por Zavaleta, con algunos intentos de ampliar su uso hacia la contemporaneidad, y de ahí proponemos la necesidad de "indianizar" el poder dual, enfocándonos en los momentos pasados de rebeliones indígenas y de intentos de construir su propio poder. Con esta base, sostenemos que la llegada de Evo Morales Ayma a la presidencia en 2006 podría haber sido un primer signo de convergencia de dos tipos de poder dual en Bolivia, uno clasista y otro indianista. En la sección posterior, reinterpretamos el desarrollo del gobierno de Carlos Mesa de 2003 a 2005 con este enfoque, y también el proceso político del gobierno de Evo Morales. Argumentamos que la figura política de Carlos Mesa funciona como "espectro" (fantasma) recurrente del nacionalismo revolucionario, del cual depende el gobierno de Evo Morales y del cual difícilmente se escapa (cf. Derrida, 1996). Siguen las reflexiones finales.

\section{El concepto de "poder dual"}

\begin{abstract}
[L] a cuestión de la dualidad de poderes es quizá una de las más enjundiosas en lo que se refiere a la construcción permanente de la teoría marxista del Estado
\end{abstract}

(Zavaleta, 2012 [1973]: 375).

\section{Poder dual según René Zavaleta Mercado}

En los estudios del pensamiento de René Zavaleta Mercado, ha sido común dividir su etapa intelectual en tres: etapa inicial de nacionalismo revolucionario, etapa media de marxismo ortodoxo y etapa tardía de marxismo heterodoxo (Antezana, 1991). El tema en el cual nos enfocamos aquí, la cuestión de poder dual, correspondería según este esquema a la etapa media, con la publicación de El poder dual en América Latina (Zavaleta, 2012 [1973]). Sin embargo, fue Gil (2016) quien señaló el carácter heterodoxo en la argumentación de Zavaleta sobre este

\footnotetext{
${ }^{3}$ Existe un temprano intento de reflexionar en esta misma línea (Huaca Bustamante, 2016), lo que nos da aliento para continuar con los argumentos que siguen.
}

EntreDiversidades. Revista de Ciencias Sociales y Humanidades, Vol. 9, Núm. 1 (18), enero-junio 2022. Páginas: 360-381 ISSN-e: 2007-7610. https://doi.org/10.31644/ED.V9.N1.2022.A15 
tema, y la continuación de ciertas temáticas, sobre todo históricas, en sus escritos posteriores (2016: ver sobre todo el capítulo III). ${ }^{4}$ Siguiendo esta línea de pensamiento, con base en una ponencia previa (Fujita, 2012), argumentamos que esta etapa de Zavaleta, de pensar sobre el poder dual, figura como precursora importante a sus reflexiones posteriores y tardías sobre la diversidad social en Bolivia, es decir, sobre su "abigarramiento", 5 aunque todavía no la toma en cuenta plenamente, por lo que se requiere trazar unas líneas complementarias para establecer esta continuidad. Asimismo, dado que el interés de Zavaleta en la cuestión del poder dual se remonta por lo menos a 1962, cuando se publica su artículo "La Revolución Boliviana y el doble poder" (Zavaleta, 2012 [1962]), año que se situaría en plena etapa inicial de sus escritos, podría señalarse que este tema subyace como telón de fondo atravesando todas las etapas de su pensamiento.

El concepto de poder dual tiene su origen en la revolución rusa, y emerge con la posterior teorización de Lenin. Según esta perspectiva, en el proceso revolucionario ruso hubo un entrecruzamiento de la revolución burguesa con la posterior revolución socialista, o sea, existió una fase de tránsito de movilizaciones democráticas a revoluciones socialistas. "Son [...] dos poderes, dos tipos de Estado que se desarrollan de un modo coetáneo en el interior de los mismos elementos esenciales anteriores; su sola unidad es una contradicción o incompatibilidad (en su forma intensificada, es decir, su antagonismo)" (Zavaleta, 2012 [1962]: 378). De ahí surge la pregunta sobre cómo transformar las movilizaciones democráticas (de la burguesía) en revoluciones socialistas (de obreros o masas). De no realizar plenamente esto, queda una situación inconclusa en la que los dos poderes coexisten durante un período prolongado (o permanentemente), y el sector obrero/masas más bien se acerca al polo burgués asumiendo esta posición como la suya. Se puede extender, argumenta Zavaleta, esta cuestión hacia los procesos revolucionarios de América Latina del siglo XX. Cabe enfatizar que cuando Zavaleta habla de la autonomía efectiva de la clase obrera, es sobre todo en el nivel ideológico ("El sujeto debe existir antes que su poder", 2012 [1962]: 408). Teniendo en cuenta esto nos preguntamos, ¿la llegada del MAS de Evo Morales al gobierno en 2016 no ha sido otro caso del poder dual, pero con nuevos protagonistas? Si es así, ¿cómo podemos caracterizar la dualidad de poder en el desarrollo de su gobierno? Pero antes de abordar estas cuestiones, primero sigamos con la clarificación y extensión conceptual.

El mismo Zavaleta, al parecer, se mostraba dubitativo sobre hasta qué punto era generalizable esta noción de poder dual. Si suponemos que "la unidad del poder es connatural al Estado moderno", la dualidad de poderes no es solo una situación anómala, sino también una designación anómala (2012 [1962]: 377). Siendo la noción de "autonomía” clave en el pensamiento zavaletiano, y con un énfasis en que "[n]o hay autonomía donde no hay unidad” (2012 [1962]: 377), señala que "la dualidad de poderes es una anomalía o enfermedad que se presenta en el seno del poder del estado" (2012 [1962]: 378). Sin embargo, el concepto ha seguido siendo retomado, no solo por Zavaleta en su momento sino también por otros investigadores posteriores (véase Shirai, 2007).

\footnotetext{
${ }^{4}$ Antes del trabajo mencionado de Mauricio Gil (2016), podemos decir que esta etapa de la teorización del poder dual se había quedado en el olvido. Tanto en Antezana (1991) como en Tapia (2002), las monografías representativas del estudio sobre Zavaleta no dan casi ningún tratamiento de esta obra.

${ }^{5}$ Sobre esta etapa tardía de Zavaleta, los trabajos mencionados en la nota al pie anterior de Antezana (1991) y Tapia (2002) siguen siendo la mejor introducción y estudio del tema.
}

EntreDiversidades. Revista de Ciencias Sociales y Humanidades, Vol. 9, Núm. 1 (18), enero-junio 2022. Páginas: 360-381 ISSN-e: 2007-7610. https://doi.org/10.31644/ED.V9.N1.2022.A15 
Carlos Mesa, con quien nos detendremos más adelante con su gobierno de 2003 a 2005, en su carrera anterior como periodista, había realizado una entrevista a Zavaleta en 1983, en la que vuelve insistentemente a esta noción de poder dual. En la grabación de la misma, que es hoy accesible a través de YouTube ("Entrevista de Carlos Mesa a René Zavaleta", ver Laguna, 2019a, 2019b y 2019c), hablan de la vigencia del nacionalismo revolucionario en la Bolivia post- democratización del 1982. En una entrevista de casi 45 minutos, Mesa vuelve dos veces al tema del poder dual y el cogobierno, como si esto fuera la cuestión clave al reflexionar sobre los legados de la revolución del 52. Primero, se refiere a la opinión de Walter Guevara Arce sobre si la dualidad de poderes que existió no llegó a la pérdida de poder de decisión por parte del gobierno revolucionario. Segundo, apunta al entonces reciente pedido de cogobierno por parte de la Central Obrera Boliviana (COB) en el gobierno de Hernán Siles Zuazo — que llegó al poder en 1982 - y pregunta si esto no era la forma de superar el proceso democrático tradicional y dar un paso necesario adelante en la modernización de Bolivia. A estas preguntas, Zavaleta responde repitiendo su postura de que la dualidad de poderes o el cogobierno es una contradicción de términos que no puede ser propuesta como un régimen estable, y que la noción de cogestión es más apropiada porque es compatible con cualquier régimen democrático. Sin embargo, nos preguntamos, ¿no será la cogestión una versión degradada de poder dual?, y, ¿̨no está esta respuesta de Zavaleta demasiado centrada en la coyuntura de democratización del 1982? Se nota que Mesa buscaba entonces abrir las posibilidades no explotadas de la revolución del 52. Nos hace recordar que el concepto de la sociedad "abigarrada”, como una característica fundamental de la sociedad boliviana, sigue siendo sujeto a un debate similar; si el abigarramiento es algo anómalo y patético o si más bien cuenta con una posibilidad esperanzadora y emancipadora. ${ }^{6}$

Zavaleta encuentra dos momentos clave de dualidad de poderes en la historia contemporánea de Bolivia: la revolución ("Revolución Nacional") de 1952 y el gobierno de Juan José Torres de 1970 a 1971.7 En la revolución del 52, inmediatamente después de las insurrecciones y en el período inicial de la revolución, existía "una hegemonía de la clase obrera" (Zavaleta, 2012 [1973]: 416), donde "[l]a asamblea sindical se convirtió en la suprema ley y la suprema autoridad” (2012 [1973]: 417). Califica a la clase obrera boliviana de esta época como "dotada de mejor sentido histórico que todas sus iguales en el continente” (2012 [1973]: 417). Sin embargo, debido a la falta de conciencia organizada por parte de los mineros, y sin poder constituirse como un partido vanguardista, rápidamente se disipan para someterse bajo el polo burgués — representado por el partido gobernante Movimiento Nacionalista Revolucionario (MNR) y las políticas sugeridas del gobierno de Estados Unidos-. Zavaleta considera que el cogobierno MNR-COB constituyó una degeneración del embrión de la dualidad de poderes, que solo sirvió para consagrar el poder de Juan Lechín, el líder de la COB (2012 [1973]: 420). La misma fuerza obrera — casi veinte años después, en el gobierno militar izquierdista de Juan José Torres de 1970 a 1971 - llega a formar la Asamblea Popular, la máxima instancia de la toma de decisiones. Sin

\footnotetext{
${ }^{6}$ En el congreso bi-anual de la Asociación de Estudios Bolivianos de 2015, estuve participando en un debate acalorado sobre este tema con Silvia Rivera Cusicanqui y Luis Tapia.

${ }^{7}$ La redacción de este párrafo se inspiró en las ideas de Oliver Costilla (2016), además de las obras de Zavaleta mencionadas arriba.
}

EntreDiversidades. Revista de Ciencias Sociales y Humanidades, Vol. 9, Núm. 1 (18), enero-junio 2022. Páginas: 360-381 ISSN-e: 2007-7610. https://doi.org/10.31644/ED.V9.N1.2022.A15 
embargo, la Asamblea careció de poder de coerción y del respaldo de los generales, quienes eran esta vez la representación del polo burgués. Al final, esta posibilidad se queda sin desarrollar plenamente.

Esto nos hace recordar el argumento en Miyachi (2014) sobre los movimientos indígenas contemporáneos de Bolivia y Ecuador que señala que combinan una estrategia de toma de poder intra-institucional (i.e. a través de las elecciones) con una extra-institucional (i.e. a través de bloqueos, paros, y asambleas), y que en 1992, en el movimiento en contra de la conmemoración del quinto centenario de la llegada de Cristóbal Colón a las Américas, la Confederación Sindical Única de Trabajadores Campesinos de Bolivia (CSUTCB) realiza un llamado para establecer una Asamblea de Naciones Originarias, sin llegar a tener un poder efectivo ni una continuidad posterior (2014: ver sobre todo el Capítulo 2). Aquí la historia parece repetirse más allá de lo vislumbrado en las obras zavaletianas y más allá del ámbito obrero, y parece señalar la necesidad de ampliar el horizonte hacia los movimientos indígenas. Zavaleta, como se puede ver también en la entrevista de Carlos Mesa arriba mencionada, se mostró en contra de expandir esta noción de la dualidad de poderes, diciendo que "él [Trotsky] mismo extendió tanto el significado de la dualidad de poderes como metáfora o figura de la teoría marxista del Estado que la volvió apta para comprender cualquier sociedad. Con un brillo característico, acabó por diluir un concepto que era muy preciso en Lenin" (Zavaleta. 2012 [1973]: 405). Sin embargo, en una sociedad como la boliviana, donde las fisuras profundas en la estructura social llegan a formar una sociedad altamente conflictiva, ¿no será cierto que la dualidad de poderes es mucho más extensa que solo en los momentos críticos de la "revolución" tradicional marxista, apareciendo más bien en cada insurrección indígena, incluso presente en cada momento y cada ámbito de la sociedad?

En la contemporaneidad, Gray Molina (2007) reinterpreta esta noción de poderes duales como el compartir del poder entre el sector obrero-campesino y el gobierno revolucionario del MNR, lo que posibilita asegurar el apoyo político y la gobernabilidad. Extiende la noción de poder dual para situar la reforma descentralizadora de Participación Popular de 1990 en Bolivia bajo el primer gobierno de Gonzalo Sánchez Lozada (1993-1997), y el establecimiento del mecanismo de control social en dicha reforma, como un ejercicio de poder dual (2007: 24). Según Gray, esto forma parte de un conjunto de mecanismos políticos que en Bolivia asegura una relativa paz en medio de una desigualdad abismal, o, en otras palabras, la "armonía de desigualdades" (2007: 26-28). ${ }^{8}$ Ahora bien, este argumento de Gray puede ser visto como demasiado fácil y optimista, que no toma en cuenta el complicado proceso de los primeros años de la revolución de 52, además proponiendo la sociedad boliviana como un caso ejemplar en una situación abismal de desigualdad. Sin embargo, Gray comparte con Mesa (ver arriba) una esperanza todavía no fundamentada sobre que la dualidad de poderes puede ofrecer una salida a la compleja sociedad plagada de colonialismo y desigualdad. También amplía esta noción zavaletiana para intentar ver un proceso de adquisición y construcción del poder en la sociedad, es decir, fuera del Estado. Si aceptamos que esta reforma de Participación Popular preparó la base sociopolítica en las regiones para las fuertes movilizaciones sociales que comienzan desde el 2000

${ }^{8}$ El término "armonía de desigualdades" proviene de Irurozqui (1994).

EntreDiversidades. Revista de Ciencias Sociales y Humanidades, Vol. 9, Núm. 1 (18), enero-junio 2022. Páginas: $360-381$ ISSN-e: 2007-7610. https://doi.org/10.31644/ED.V9.N1.2022.A15 
(Blanes, 2000), que culmina en la llegada del Movimiento al Socialismo (MAS) y Evo Morales al poder en 2006 (elecciones generales de 2005), parece convencernos de la necesidad de ampliar la noción de poderes a esferas fuera del Estado formal.

Curiosamente, este interés en la dualidad de poderes y cogobierno es compartido por Luis Tapia, quien, como vimos arriba, en su estudio comprehensivo sobre el pensamiento de Zavaleta Mercado (Tapia, 2002), casi no presta atención a la "etapa intermedia de marxismo ortodoxo". En 2007 publica el libro La igualdad es cogobierno (Tapia, 2007), en el que intenta avanzar su pensamiento sobre la igualdad política y arguye la necesidad de salir de la noción estrecha de democracia como representación por elecciones, proponiendo una democracia politizada y participativa y apuntando a la necesidad de "gobernar con otros" (2007: 166). Aquí, cogobierno implica la descentralización del poder (2007: 167). Tapia enfatiza este aspecto de la siguiente manera: "Considero que el objetivo de la igualdad social, que es un ideal socialista, en las condiciones multiculturales y semicoloniales todavía, de países como Bolivia, implica que la democratización necesita pasar por un rediseño o refundación multicultural de las formas de gobierno común" (2007: 172).

Notemos aquí su énfasis en la pluralidad de "formas de gobierno". Esto requeriría el reconocimiento de la pluralidad de poderes en una sociedad "multisocietal" como la boliviana, y una refundación del Estado para posibilitar el cogobierno, o quizás, yendo un paso más adelante del término empleado por Tapia, el pluri-gobierno. Una necesidad de complejizar la institucionalidad política.

\section{Historicidad del poder dual más allá del marxismo: hacia una “indianización” del concepto}

A pesar de la insistencia de René Zavaleta Mercado en no ampliar el alcance del concepto de poder dual, la discusión en el anterior apartado nos lleva a proponer la necesidad de extender la noción de la dualidad de poderes más allá de la etapa y la perspectiva marxistas, hacia los diversos momentos en la historia larga de las rebeliones indígenas, donde se reclama la "soberanía indígena con leyes propias" (Hylton, et al., 2003: 5). No es posible realizar aquí una pesquisa exhaustiva sobre este tema, pero enfocarnos en los resultados de investigaciones recientes nos ayuda a re-pensar esta cuestión de poderes.

Tomaremos en cuenta la historia de las diversas sociedades indígenas de Bolivia. Es importante destacar primero que el proceso de construcción de poder no se limita a los momentos culminantes de rebeliones (o revoluciones), y que más bien ha sido algo institucionalizado a través del tiempo, construyéndose mediante prácticas y negociaciones cotidianas. Como Platt (2016) ha señalado en su libro ya clásico Estado boliviano y ayllu andino para la región de Norte de Potosí, después de la reforma Toledana del siglo XVI ha existido lo que se llama un "pacto de reciprocidad" entre la parte espańola/colonial/republicana (Estado) y la parte indígena (ayllu). "La esencia de este pacto consistía en la obligación del Estado no solo de reconocer los derechos colectivos de los ayllus a sus tierras, sino también de aceptar como contraparte los servicios tradicionales y la tasa, antiguo tributo indígena pagado por los indios" (2016: 62).

EntreDiversidades. Revista de Ciencias Sociales y Humanidades, Vol. 9, Núm. 1 (18), enero-junio 2022. Páginas: 360-381 ISSN-e: 2007-7610. https://doi.org/10.31644/ED.V9.N1.2022.A15 
Es decir, siempre ha existido una suerte de autogobierno por parte de las sociedades indígenas. Como enfatiza Rivera Cusicanqui $(2016,2010)$ en su lectura crítica pero simpática de la obra de Platt, este pacto de reciprocidad nunca fue equitativo, sino uno que se ha ido degradando a través del tiempo por el violento colonialismo interno. La misma Rivera Cusicanqui (2010) intenta expandir la noción de este pacto hacia las otras regiones andinas de Bolivia en su ensayo interpretativo de la historia boliviana de larga duración de colonialismo y de memorias prehispánicas, combinada con la memoria media de la historia post-independencia y el ciclo corto de la historia después de la revolución del 1952. Este ensayo nos ofrece las pautas para ampliar las reflexiones teóricas de René Zavaleta Mercado más allá del ciclo corto de post-1952, una tarea en la que el mismo Zavaleta emprendió hacia la etapa final de su vida intelectual, pero sin lograr concretizar.

Esta perspectiva de un pacto de reciprocidad pero desigual tiene eco contemporáneo desde una perspectiva estado-céntrica en el Informe Nacional sobre Desarrollo Humano 2007. El estado del Estado en Bolivia, publicado por la oficina boliviana de Programa de Naciones Unidas para el Desarrollo (PNUD Bolivia, 2007), en el cual el coordinador principal del informe fue George Gray Molina. Aquí introduce el concepto de un Estado "con huecos", que es descrito de la siguiente manera en el resumen de este informe:

El Estado boliviano se asemeja a un Estado "con huecos" por la accidentada extensión territorial de su autoridad burocrática y legal. En los "huecos", se negocia la autoridad legal estatal con organizaciones sociales, indígenas, locales y regionales. El Estado "con huecos" tuvo capacidad de adaptarse a balances de poder existentes —identidades locales, regionales, sociales, y étnicaspero no ha logrado construir un espacio público común de autoridad, legitimidad y soberanía estatal. Por ello subsiste un arraigado pluralismo institucional (2007: diapositivas 9 y 10).

En la sociedad boliviana actual se puede presenciar este proceso de negociación cuando, en medio de conflictos sociales, los agentes del Estado (policías y jueces) son expulsados de las regiones con fuerte presencia indígena. La continuación del gobierno de Evo Morales durante trece años (2006-2019), a pesar de aumentar la presencia del Estado en las diferentes esferas de la sociedad boliviana, parece no haber cambiado esta condición fundamental. Dada esta realidad, es difícil sostener una teoría del poder como monopolio del Estado ni argumentar que la dualidad de poderes es algo limitado a los momentos excepcionales del proceso revolucionario. Es un proceso de constante y cotidiana negociación de poderes, y en este sentido se ve la necesidad de generalizar el concepto de poder dual más allá de los momentos excepcionales hacia una condición sostenida bajo la situación colonial y la resistencia de las sociedades indígenas.

Sin embargo, es en momentos de rebeliones indígenas en los cuales los diversos proyectos y las estrategias de las sociedades indígenas salen a luz. En este sentido cobra importancia "la crisis como método de conocimiento" (Zavaleta, 2013 [1983]: 104-107; Antezana, 2009). Asimismo, la noción del "pacto" puede dar una impresión de que la dualidad de poderes que está en juego aquí consiste en dos polos distintos, el Estado y las sociedades indígenas en conflicto y negociación, y que no se trata de dos fuerzas compitiendo por el mismo poder estatal. Sin

EntreDiversidades. Revista de Ciencias Sociales y Humanidades, Vol. 9, Núm. 1 (18), enero-junio 2022. Páginas: 360-381 ISSN-e: 2007-7610. https://doi.org/10.31644/ED.V9.N1.2022.A15 
embargo, las investigaciones recientes sobre los diversos momentos de rebeliones indígenas nos demuestran que es precisamente en esos momentos rebeldes que la competencia entre los dos poderes se asemeja a la dualidad de poderes explicada arriba.

En el trabajo sobre el ciclo rebelde liderado por Túpaq Katari del siglo XVIII, Thomson (2003, 2006) se enfoca en las visiones políticas expresadas por los aymaras. Ahí señala que los españoles que se rindieron en Sorata fueron perdonados pero también obligados a vestirse como los indios, akhullicar ("mascar" la coca), andar descalzados y llamarse como qullas (denominación de la gente que habita el altiplano surandino empleada por los incas). En el levantamiento del pueblo de Caquiaviri en 1771, los vecinos del pueblo se vieron obligados a pronunciar públicamente que pertenecían a, y vivían en, un ayllu. Todo esto indica que los aymaras tenían su propio proyecto político indígena y que trataron de posicionar a los españoles como una unidad dentro de un nuevo régimen político liderado por los indígenas.

Hylton (2004) también señala que en la guerra federal de 1899 los federalistas insurgentes tuvieron un proyecto de federalismo aymara-quechua basado en: "autogobierno, manejo comunal de tierras y cese de todo tipo de impuestos que caían sobre los hombros de los comunarios" (2004: 112), con el cual "la república se hubiera transformado de una manera revolucionaria para la época” (2004: 114). Reitera la importancia de verlo como un proyecto político propio, no asimilable a una corriente política occidental:

Si eran "liberales", lo eran a su manera, negando los componentes básicos del liberalismo: el individuo, la delegación de la representación política a través de partidos y la propiedad privada. Si buscaban "ciudadanía", ésta la defendían a su manera, reclamando respeto a la propiedad comunal (antitética al liberalismo clásico), la abolición de impuestos neocoloniales como la contribución indigenal y el gobierno indígena en los niveles local y regional (2004: 112).

Asimismo, Hylton (2003) señala que en la rebelión en Chayanta de 1921 los insurgentes indígenas buscaron activamente una alianza con los obreros y los intelectuales radicales urbanos. Aquí se puede ver que, más que un polo opuesto al estado de separatismo cerrado, las rebeliones indígenas se dedican a un activo proceso de construcción de poder que se vincula con los otros sectores de la sociedad. Aunque Hylton (2004) considera a las acciones contemporáneas de Felipe Quispe, el Mallku, como una posición de separatismo basado en el nacionalismo aymara, se puede seńalar que él estuvo también en alianza con otros grupos sociales y organizaciones en los diversos momentos de su activismo.

Estas consideraciones nos lleva a concluir provisionalmente que existe la necesidad de re-articular y re-contextualizar los conceptos marxistas en un ciclo más largo de movilizaciones sociales en Bolivia. Esto sería una tarea hacia la "indianización”, es decir, desde una perspectiva indianista (Reinaga, 2010), de la etapa marxista de Zavaleta. Así, el auge del katarismo, el activismo de Felipe Quispe, y la llegada a la presidencia de Evo Morales y el MAS pueden ser interpretados como un nuevo ciclo de construcción de poder indígena, permitiéndonos analizar la dinámica socio-política contemporánea a través de la perspectiva de la dualidad de poderes pero ampliada.

EntreDiversidades. Revista de Ciencias Sociales y Humanidades, Vol. 9, Núm. 1 (18), enero-junio 2022. Páginas: 360-381 ISSN-e: 2007-7610. https://doi.org/10.31644/ED.V9.N1.2022.A15 


\title{
La política boliviana del siglo XXI y el fantasma del nacionalismo revolucionario
}

\author{
Siguiendo rutas diversas, el MAS articuló las demandas de ambas "agendas" \\ en torno al nacionalismo y al indigenismo y el conjunto de los discursos politicos \\ se ordena por afinidad o cuestionamiento en torno a estos ejes \\ (Mayorga y Rodríguez 2013: 98).
}

\section{Octubre de 2003 y el gobierno de Carlos Mesa}

Con la renuncia y salida del país del entonces presidente Gonzalo Sánchez de Losada el 17 de octubre de 2003 — tras una masiva protesta social en contra del proyecto de exportación de gas natural por un puerto chileno y de las medidas represivas del gobierno hacia los sectores sociales-, el vicepresidente Carlos Mesa Gisbert asumió la presidencia. El gobierno de Carlos Mesa, de octubre 2003 a junio 2005, tomó las demandas de los movimientos sociales como su agenda política: (1) realización de un referéndum sobre las políticas hidrocarburíferas, (2) una nueva ley de hidrocarburos reflejando el resultado del referéndum, y (3) convocar a asamblea constituyente para definir una nueva constitución. Esto, la llamada "agenda de octubre", ya combinaba los elementos de nacionalismo (nacionalismo de recursos) y aspiraciones plurinacionales que Mayorga y Rodríguez (2013) señalan. El gobierno de Mesa avanzó en algunas reformas políticas importantes - introducción del referéndum como herramienta formal y realización del mismo sobre hidrocarburos, modificación de la ley electoral para permitir a asociaciones ciudadanas y organizaciones indígenas como base sobre la cual se podría candidatear en las elecciones e introducción de la meritocracia basada en una carrera burocrática estatal—, pero se estancó en medio de la creciente presión externa y por las fuerzas sociales internas que demandaron una postura firme por parte del gobierno sobre la política de recursos naturales.

Lo que se puede destacar sobre esta etapa de la política boliviana es la sorprendente cercanía entre Carlos Mesa y Evo Morales. Aunque Mesa en su memoria de gobierno (2008) niega haber tenido cualquier tipo de acuerdo con Morales y su partido MAS (Movimiento al Socialismo) (Mesa, 2008: 185), daba la impresión de que hubo un (tácito) acuerdo de gobernabilidad. El mismo Mesa relata lo siguiente:

Al principio el MAS apostó por la estabilidad y no se sumó a ninguna movilización, marcha o bloqueo. Tenía una percepción de que el escenario político nuevo les daba interesantes oportunidades y que debía evitar una desestabilización del gobierno desde la derecha y la extrema izquierda, mientras se preparaba para el futuro mediato (2008: 186).

Podemos señalar, además de la estrategia de Morales y del MAS, una cierta afinidad en las posiciones políticas de ambos políticos. Es evidente la simpatía que Mesa tiene hacia el nacionalismo revolucionario del 52, y además de estar cerca del partido que protagonizó dicha

EntreDiversidades. Revista de Ciencias Sociales y Humanidades, Vol. 9, Núm. 1 (18), enero-junio 2022. Páginas: 360-381 ISSN-e: 2007-7610. https://doi.org/10.31644/ED.V9.N1.2022.A15 
revolución —el MNR (Movimiento Nacionalista Revolucionario)—, siguió propugnando la idea del mestizaje - la ideología social principal del 52 - bajo el gobierno de Morales (Mesa, 2013). La posición de Evo Morales, aun asumiendo el rol simbólico de un líder indígena, no fue indianista. Como señala Canessa (2006), mientras Felipe Quispe Huanca mantuvo una postura estrictamente indianista, restringiendo a todos los candidatos de su partido político MIP (Movimiento Indígena Pachakuti) a la gente indígena, la manifestación identitaria indígena de Evo Morales ha sido mucho más "ligera", acercándose a la clase media urbana e incluyéndola como su base de apoyo (2006: 249-255). La posición política de Morales ha sido mucho más nacionalista que la de Quispe.

Con todo, podemos decir que tanto Carlos Mesa como Evo Morales casi simultáneamente llegaron a mandar en el país, lo que se asemejaría a una situación de dualidad de poderes. Sin embargo, aunque la llegada al poder de Evo Morales fue un momento significativo y simbólico en la historia de la política boliviana, su elección como presidente no fue tanto una estrategia activa, sino una de necesidad para re-acomodarse rápidamente después de la temprana salida de Mesa en 2005. Podríamos añadir que no fue tanto el MAS como partido sino los movimientos indígenas y sociales del 2003 los que cambiaron el curso de la política e introdujeron una nueva matriz plurinacional y/o indianista en el ámbito del poder de Estado.

\section{La asamblea constituyente 2006-2009 y las elecciones generales del 2009}

El gobierno de Evo Morales logró lanzar la convocatoria de Asamblea Constituyente, algo que el gobierno de Mesa no consiguió realizar. Las actividades de la misma instancia empezaron el 6 de agosto de 2006. El proceso hasta la aprobación de la nueva constitución en enero de 2009 a través del referéndum constitucional, fue un proceso altamente conflictivo, y para las organizaciones indígenas un proceso angustioso. Schavelzon (2012) describe detalladamente este proceso: además del conflicto entre las "dos Bolivias" (Bolivia blanca y Bolivia indígena), hubo un difícil conflicto entre el partido oficialista MAS y las organizaciones indígenas. Schavelzon caracteriza la constitución redactada como una constitución "abierta":

La Constitución abierta, ambigua, indefinida, o con conceptos abarcativos es producto en primer lugar de la delicada correlación de fuerzas entre el MAS y la oposición, que obligaba por su propio peso a que sobre la base del texto del MAS, PODEMOS y el resto de la oposición pudieran intervenir en la redacción (2012: 441).

El tema de las circunscripciones especiales indígenas y su reducción al final a solo siete escaños en todo el ámbito nacional presentó un dilema difícil para el CONAMAQ (Consejo Nacional de Ayllus y Markas de Qullasuyu) y la CIDOB (Confederación de Pueblos Indígenas del Oriente Boliviano), organizaciones que preciesamente planteaban estas reformas. Esto, a pesar de que fueron reconocidos más de 30 naciones y pueblos indígenas originarios campesinos, incluyendo al pueblo afroboliviano. El MAS mostró un fuerte afán para mantener su hegemonía como partido, y rechazó la idea de modificar las jurisdicciones existentes en el régimen autonómico, negando o minimizando estas reformas. Debido al apoyo masivo al MAS en el occidente boliviano y a la

EntreDiversidades. Revista de Ciencias Sociales y Humanidades, Vol. 9, Núm. 1 (18), enero-junio 2022. Páginas: 360-381 ISSN-e: 2007-7610. https://doi.org/10.31644/ED.V9.N1.2022.A15 
presión tácita de tener que apoyar a un gobierno indígena, estas dos organizaciones se encuentran en una posición bastante difícil al no poder exigir un cambio radical en el texto de la constitución. Aquí, ya podemos ver un proceso en el que el MAS, más que un partido que representa a las organizaciones indígenas, intenta contener el poder de estas para hegemonizar su propio poder. Mientras los indígenas ocupan algunos puestos del Estado boliviano simbólicamente importantes - Ministerio de Relaciones Exteriores y la Academia Diplomática Plurinacional, Viceministerio de Descolonización en el Ministerio de Cultura-, en el proceso constituyente quedan excluidos del proceso de la "refundación" del Estado boliviano. Nos parece que esto se asemeja de manera considerable a la situación de la dualidad de poderes que los obreros experimentaron en el gobierno del MNR después de la revolución de 1952.

Si pensamos en el proceso de descolonización como el trazar de un nuevo mapa, es decir, una nueva cartografía del Estado (Arnold y Villarroel, 2004; Gustafson, 2009), la nueva constitución boliviana de 2009 quedó lejos de esta meta. Sin embargo, el MAS logra aprobar esta constitución "abierta" con el 61.4\% de apoyo. Con este logro, el partido logra desmontar el foco opositor del oriente boliviano - los empresarios y la Unión Juvenil Cruceñista - en las elecciones generales del 6 de diciembre del mismo año, y Evo Morales obtiene el 63.9\% de los votos, una cifra aún más alta que la obtenida en las elecciones generales de 2005. Así, podemos ver que al optar por, y priorizar, una posición nacionalista y centralista, Evo Morales y su partido MAS obtienen un nivel de aprobación histórica. Este proceso constituyente cimentó el camino que la política boliviana pretende seguir en la próxima década, comenzando con las convulsionadas elecciones generales del 2019, cuando Evo Morales no logra obtener una mayoría cómoda, y tras un alegato de fraude electoral y protestas sociales es sugerido por las Fuerzas Armadas a renunciar.?

\section{El conflicto del TIPNIS y el de la salida al mar}

En la década de 2010, el gobierno del MAS se caracteriza más por una creciente tendencia de intentar acallar con fuerza las movilizaciones sociales y de protesta. Esto se combina con una postura de desarrollismo tradicional del estilo del 52, con un fuerte énfasis en la integración del espacio nacional con carreteras. Todo ello desemboca en el conflicto en torno al TIPNIS (Territorio Indígena y Parque Nacional Isiboro-Securé), cuando en 2010 el gobierno anuncia el proyecto de construcción de una carretera que atravesaría este territorio (Marston y Kennemore, 2019; Rivera Cusicanqui, 2015; Chávez, et al., 2012). Una marcha de los pueblos del oriente, liderada por la CIDOB en 2011, respaldada por el CONAMAQ y con un masivo apoyo de la clase media urbana, genera una pérdida considerable de votos del MAS en las elecciones judiciales del mismo año, lo que obliga al gobierno a congelar el proyecto y a anunciar un proceso de consulta previa con la población de las áreas afectadas. Como señalan los estudios mencionados, este conflicto demostró, por un lado, una solidaridad entre los sectores indígenas andinos y de las tierras bajas, y, por otro lado, sacó a luz la historia de la migración del sector cocalero hacia las tierras del TIPNIS (orientada a actividades madereras que generan deforestación), así como la

\footnotetext{
${ }^{9}$ Cabe señalar aquí que este artículo no señala que hubo fraude en las elecciones generales de 2019, de hecho, los estudios posteriores no encuentran ninguna prueba de esta. También las masacres que ocurrieron después de la renuncia de Morales son una seria violación de derechos humanos.
}

EntreDiversidades. Revista de Ciencias Sociales y Humanidades, Vol. 9, Núm. 1 (18), enero-junio 2022. Páginas: 360-381 ISSN-e: 2007-7610. https://doi.org/10.31644/ED.V9.N1.2022.A15 
subordinación de la población indígena de tierras bajas por parte de estos migrantes. En los años siguientes, el gobierno de Morales intentaría en varias ocasiones más obtener la aprobación del proyecto. El gobierno trató de dividir a la CIDOB y al CONAMAQ para establecer organizaciones paralelas con posturas afines a la del oficialismo.

A través de este conflicto, comienzan a aparecer opiniones señalando el "giro a la derecha" del gobierno de Morales (Rivera Cusicanqui, 2015), detectando una postura colonialista (Puente, 2017) o neocolonialista (Mamani, 2017). A pesar de lograr la incorporación de un sector previamente excluido de la política, la postura del gobierno del MAS ya se alejaba considerablemente de lo que al comienzo se denominó como la "tercera revolución boliviana". También la insistencia en el proyecto por parte del MAS lo llevó a una pérdida considerable de votos en las elecciones subnacionales de 2015 , obteniendo solo el $41.8 \%$ de los votos. ${ }^{10}$ En este momento es cuando parece que el gobierno del MAS definitivamente se aparta de los indígenas de la sociedad boliviana, si no es que de todos los indígenas y de los sectores sociales. Esto nos indica la importancia del proceso de la construcción del poder local y regional indígena, más allá de la lucha por llegar a la hegemonía y mantenerla en el ámbito estatal.

Otro acontecimiento que nos parece importante en esta época de la política boliviana es la disputa internacional con Chile sobre la salida soberana de Bolivia al mar, llevada ante la Corte Internacional de Justicia de La Haya. En 2014, el gobierno de Morales asigna a Carlos Mesa Gisbert como vocero de la causa marítima, y a Eduardo Rodríguez Veltzé — quien asumió las funciones del presidente interino después de la renuncia de Mesa en 2005 para convocar las elecciones generales - como agente ante la Corte de La Haya. En este proceso, Morales crea una red de expresidentes que no solo incluye a Mesa y Rodríguez Veltzé, sino a Jorge Quiroga (2001-2002) y a Jaime Paz Zamora (1989-1993). Aunque el fallo final de la corte en 2018 no favoreció a Bolivia, a través de este conflicto Evo Morales se posiciona como el heredero de la presidencia republicana, situándose firmemente como nacionalista. Además, Carlos Mesa resucita como figura política, con su postura firmemente nacionalista y colaborador del gobierno boliviano en este tema marítimo. Una vez más se ve un acercamiento entre Evo Morales y Carlos Mesa, y el nacionalismo es el factor que une a estos dos personajes políticos.

\section{La debacle de las elecciones generales de 2019}

Es en medio de una creciente tensión social y una insatisfacción con el gobierno de Evo Morales que Bolivia llega a sus elecciones generales de 2019. ${ }^{11} \mathrm{Y}$ es aquí cuando Carlos Mesa toma la

\footnotetext{
${ }^{10}$ Sin embargo, algunos analistas señalan que el MAS siempre tuvo dificultades en las elecciones subnacionales, mientras ganaba cómodamente en las nacionales, lo que puede reflejar un delicado y equilibrado cálculo del electorado boliviano.

${ }^{11}$ Como uno de los primeros estudios que trata de dar sentido a esta etapa de la política boliviana, ver Valdivia (ed.) (2021). Nuestra visión en este artículo está en línea con la interpretación ofrecida en ese trabajo. Lamentamos no poder citar sistemáticamente los trabajos publicados dentro de Bolivia debido a no haber podido realizar trabajos de campo en el país por la situación de la covid-19. A pesar de lo que se argumenta en este artículo, no todo se pinta negativamente en la década de los 2010 acerca de los intentos de constuir un Estado plurinacional. Umezaki (2018) narra un proceso complejo experimentado por la comunidad afroboliviana en la Asamblea Constituyente, y el siguiente trabajo de construir materiales bajo el marco de educación plurinacional.
}

EntreDiversidades. Revista de Ciencias Sociales y Humanidades, Vol. 9, Núm. 1 (18), enero-junio 2022. Páginas: 360-381 ISSN-e: 2007-7610. https://doi.org/10.31644/ED.V9.N1.2022.A15 
decisión de volver de nuevo al escenario electoral como candidato con su propia organización ciudadana. A pesar de su candidatura, y pese a las fuertes dudas de inconstitucionalidad puestas sobre la re-postulación de Evo Morales, el ambiente general en el curso del 2019 fue de una victoria anticipada de Morales. En el proceso preparatorio hacia los comicios, la figura de Carlos Mesa no fue muy visible.

Sin embargo, Mesa obtuvo un inesperado nivel de votos en la primera ronda de la elección, posicionándolo en el segundo lugar, cerca del margen que le permite entrar en la segunda vuelta con Evo Morales. Aunque hay debate sobre si realmente hubo un fraude en el cómputo de los votos, es posible que este ascenso sorpresivo de Carlos Mesa haya puesto en aprietos al oficialismo. El estrecho margen por el cual el MAS iba a evitar la segunda vuelta puso en seria duda la legitimidad del resultado de las elecciones. Evo Morales ya no recibió el nivel de votación histórico de las veces anteriores, y estas elecciones generales de 2019 pueden ser interpretadas como la segunda ronda de voto de castigo al MAS después de los comicios inmediatamente posteriores al conflicto de TIPNIS.

Parece existir una suerte de paradoja aquí. El gobierno del MAS, por acercarse al nacionalismo revolucionario tradicional, por un lado, logra establecer una hegemonía temporal, pero por otro lado, y por su actitud soberbia, invita a Carlos Mesa a reaparecer como fuerte opositor político, casi como un fantasma que regresa, y con esto permite la rearticulación de las fuerzas de extrema derecha. Esto prueba la continuada vigencia del nacionalismo revolucionario en la política boliviana. Igualmente, muestra cómo una fuerza fantasmal atrae a las nuevas fuerzas políticas, a la vez que las amenaza resucitando las figuras fantasmales que habían salido del escenario político. Podríamos decir que Carlos Mesa es también una figura fantasmal en el sentido de que aparece en momentos claves de la política boliviana del siglo XXI, pero sin una posibilidad real de "gobernar" el país.

\section{Reflexiones finales}

El proceso político de Bolivia en el siglo XXI nos muestra la continua vigencia del concepto de la dualidad de poderes de René Zavaleta Mercado. El gobierno de Carlos Mesa Gisbert (20032005) puede ser interpretado como una etapa en la que se estableció la dualidad de poderes. Quizás en esta ocasión Evo Morales efectivamente venció esta situación dual de poderes, llegando a obtener el poder con las elecciones generales de 2005. Sin embargo, en el momento de ascender a la presidencia, Morales y su partido MAS optaron por abandonar la cara indígena e indianista de su poder, y más bien adoptaron un posicionamiento burgués-tradicionalista del nacionalismo revolucionario. En este sentido, la condición difícil e inestable de la dualidad de poderes parece seguir más allá de la década de 1950 y de 1970 hasta nuestros días, y seguimos con la tarea de reflexionar el proceso político boliviano actual todavía con la ayuda del pensamiento zavaletiano.

Estas reflexiones nos indican la necesidad de complejizar el panorama teórico. Más allá de lo que ocurre en el seno del Estado, parece necesario seguir los complejos lineamientos sociales de construcción de poder tal como nos indican los recientes trabajos sobre las rebeliones indígenas históricas de Bolivia. En este proceso político boliviano del siglo XXI, también fueron

EntreDiversidades. Revista de Ciencias Sociales y Humanidades, Vol. 9, Núm. 1 (18), enero-junio 2022. Páginas: 360-381 ISSN-e: 2007-7610. https://doi.org/10.31644/ED.V9.N1.2022.A15 
los movimientos sociales activos en los primeros cinco años (2000-2005) los que marcaron la agenda política con sus visiones, aspiraciones y proyectos políticos, realizando un cambio social importante. El gobierno del MAS logró una transición de poder hacia sectores más populares, rurales e indígenas. Sin embargo, quedó pendiente la cuestión sobre precisamente qué es un gobierno indígena, plurinacional y de los movimientos sociales. El oficialismo se mostró ambiguo y se resistió a tomar una postura concreta sobre el tema.

Como el MAS se alejó cada vez más del indianismo y el plurinacionalismo, salvo en algunos sectores limitados y restringidos del gobierno boliviano, el programa de cambio político y social quedó inconcluso, y también la tarea de descolonización que por algún tiempo figuraba firmemente en la agenda del gobierno de Evo Morales. Sin embargo, el ciclo del indianismo tampoco está acabado en Bolivia, e igual que el nacionalismo revolucionario, el indianismo podría reaparecer y volver a sacudir a las fuerzas nacionalistas en un momento inesperado. Esto nos lleva a reafirmar la importancia de la constitución de un poder indígena y el desarrollo de su auto-conciencia, tal como enfatizaba Zavaleta Mercado, para la construcción real del autogobierno indígena.

Así, estamos ante la necesidad de articular en el pensamiento de René Zavaleta Mercado las discusiones del poder y la dualidad de poderes con las de la heterogeneidad social (en el término zavaletiano, "sociedad abigarrada") (Zavaleta, 2013 [1984]) e insistir en la importancia de seguir pensando en contradicciones (cf. Gudynas, 2015), más allá del afán hegemonizador y nacionalizador en el cual cayó el gobierno de Morales.

Pero esta tarea va más allá de una simple agenda izquierdista. Para una indianización de la dualidad de poderes no será suficiente una historización que vaya más allá de las etapas revolucionarias del siglo XX. Ticona (2011), refiriéndose a las propuestas educativas aymaras de Eduardo Nina Qhespi , señala que su demanda aymara de la expansión de acceso a la educación empleaba un lenguaje de hermandad, argumentando que tanto los indígenas que hablaban aymara y quechua como los españoles y mestizos hablando castellano tenían que vivir como hermanos. Es también de notar que los descendientes y escribanos del movimiento de caciques apoderados nos presentan una interpretación del colonialismo europeo como una infracción a la regla familiar. En este caso, el hombre andino es el hermano mediano (taypi) del africano, que es el mayor, y del europeo, que es el menor. El colonialismo es visto como un momento de quiebre del respeto de los tres hermanos por el hermano menor (Huanca (comp.), por publicar). El lenguaje de hermandad y de familia desmonta la dualidad o la polaridad del poder, introduciendo una dimensión ética a la discusión de poderes.

Rivera Cusicanqui (2009), basándose en una serie de conversaciones que tuvo con el yatiri-amawt'a (intelectual tradicional) Roberto Guerrero, intenta reflexionar sobre cómo la dualidad en el tejido andino, a través de la zona de contacto en el medio (taypi), llega a abrirse a una estructura territorial basada en los cuatro rincones, y cómo este movimiento posibilita una concepción espiral (cíclica pero que tiene un futuro y pasado). Este proceso de apertura y complejización, además de que nos permite explorar la dimensión ética de la constitución de este mundo, nos lleva a apreciar el valor de la espontaneidad no-coordinada de las acciones políticas en Bolivia por las fuerzas sociales (Rivera Cusicanqui y De Sousa, 2015):

EntreDiversidades. Revista de Ciencias Sociales y Humanidades, Vol. 9, Núm. 1 (18), enero-junio 2022. Páginas: 360-381 ISSN-e: 2007-7610. https://doi.org/10.31644/ED.V9.N1.2022.A15 
[Silvia Rivera Cusicanqui:] Pero de repente en 1979 los bloqueos de caminos arman una especie de consenso tácito. No necesitaron reunirse para ponerse de acuerdo, sino que espontáneamente hubo toda esa convergencia en torno a una agenda. Eso mismo ha pasado en octubre de 2003 (Guerra del Gas). Entonces pienso que hay lecturas, conocimientos no siempre verbalizados, de la situación social [...] Aquí no hay (todavía) una cultura del supermercado. Tenemos la posibilidad de enterarnos de miles de noticias en las plazas, en las calles, en los mercados. Hay todavía una fuerte cultura oral, una extraordinaria y eficaz capacidad de distribuir rumores (2015: 85).

Aquí se abre una rica oportunidad de indagar las implicaciones políticas de las narraciones orales. Serán en estos intersticios de política, ética y cultura en los que se podrán concebir las nuevas posibilidades y proyectos políticos. Seguimos con la tarea y la posibilidad de aprender del, y readaptar el pensamiento de René Zavaleta Mercado desde nuevos desafíos en los cuales nos encontramos.

\section{Bibliografía citada}

Autores varios (2007). Evo Morales entre los entornos blancoides, rearticulación de las oligarquias y movimientos indigenas. Revista Willka, Núm. 1. El Alto: Centro Andino de Estudios Estratégicos.

Antezana J., Luis H. (1991). La diversidad social en Zavaleta Mercado. La Paz, Bolivia: Centro Boliviano de Estudios Multidisciplinarios.

Antezana J., Luis H. (2006). "Veintisiete años después, el NR todavía. Entrevista por Gustavo Soto y Sergio Villena”. Decursos, Núm. 15-16, pp. 5-25.

Antezana J., Luis H. (2009). "La crisis como método en René Zavaleta Mercado". Ecuador Debate [en línea], Núm. 77, pp. 107-124. Disponible en: https://www.caapecuador.org/ecuadordebate-n-77/ (Consultado el 15 de agosto de 2021).

Arnold, Denise Y. y Villarroel, Víctor (2004). Pueblos indígenas y originarios de Bolivia: Hacia su soberania y legitimidad electoral. La Paz, Bolivia: Corte Nacional Electoral.

Blanes, José (2000). Mallkus y alcaldes. La Ley de Participación Popular en comunidades rurales del altiplano paceño. La Paz, Bolivia: Fundación PIEB (Programa de Investigación Estratégica de Bolivia).

Canessa, Andrew (2006). "Todos somos indígenas: Towards a New Language of National Political Identity”. Bulletin of Latin American Research [en línea], 25 (2), pp. 241-263. doi: https:// doi.org/10.1111/j.0261-3050.2006.00162.x (Consultado el 15 de agosto de 2021).

Chávez, Marxa, et al. (2012). Extractivismo y resistncia indigena en el TIPNIS. La Paz, Bolivia: Editorial Autodeterminación.

EntreDiversidades. Revista de Ciencias Sociales y Humanidades, Vol. 9, Núm. 1 (18), enero-junio 2022. Páginas: 360-381 ISSN-e: 2007-7610. https://doi.org/10.31644/ED.V9.N1.2022.A15 
Costilla, Lucio Oliver (2016). "René Zavaleta: la teoría en situaciones de doble poder". Aportes del Pensamiento Critico Latinoamericano [en línea], Núm. 3, pp. 99-117. Disponible en: http://iealc.sociales.uba.ar/wp-content/uploads/sites/57/2014/06/3Ren-Zavaleta-Mercado-Pensamiento-crtico-y-marxismo-abigarrado.pdf (Consultado el 15 de agosto de 2021).

Derrida, Jacques (1996). Espectros de Marx. El estado de la deuda, el trabajo del duelo y la nueva internacional. Madrid, Espańa: Trotta.

Dunkerley, James (2007). Bolivia: Revolution and the Power of History in the Present. London: Institute of Latin American Studies.

Dunkerley, James (2008-2010). "Pachakuti en Bolivia (2008-2010). Un diario personal”. Bolivian Studies Journal/Revista de Estudios Bolivianos [en línea], Vol. 15-17, pp. 9-63. doi: https:// doi.org/10.5195/bsj.2010.18 (Consultado el 15 de agosto de 2021).

Dunkerley, James (2013). “The Bolivian Revolution at 60: Politics and Historiography." Journal of Latin American Studies, 45 (2), pp. 325-350.

Fujita, Mamoru (2009). "Bolivia ni okeru 2000nendai Saha Agenda no Kentou: Senjuumin niyoru Kenryoku Kakutoku, Tasouteki Kyouzon, Genjou wo Kirihiraku Shisou” [La emergente agenda política en Bolivia de los años 2000. Toma de poder indígena, coexistencia de múltiples capas, y el pensamiento político que abre el futuro], en Murakami, Yusuke y Osonoi, Shigeo (eds.). Andesu Shokokuno Seijihendou [Los cambios políticos en los países andinos contemporáneos. En busca de gobernabilidad]. Tokio, Japón: Akashi-shoten, pp. 287-314.

Fujita, Mamoru (2012). "Las visiones políticas bolivianas post-2000 como re-lecturas de René Zavaleta Mercado. Continuidades y rupturas”. Ponencia preparada para el 6to. Congreso Latinoamericano de Ciencia Política, 14 de junio de 2012, Quito, Ecuador, también presentada en el Seminario de Posgrado en Ciencias de Desarrollo, Universidad Mayor San Andrés (CIDES-UMSA), 22 de agosto de 2012. (Documento disponible en Academia. edu, YouTube: https://youtu.be/4BsPohyQf1o).

Gil Quiroga, Mauricio (2016). Conciencia desdichada y autodeterminación de masa. En torno al pensamiento de Zavaleta Mercado. La Paz, Bolivia: Posgrado en Ciencias de Desarrollo, Universidad Mayor San Andrés (CIDES-UMSA).

Goodale, Mark (2019). A Revolution in Fragments. Traversing Scales of Justice, Ideology, and Practice in Bolivia. Durham, London, United Kingdom: Duke University Press.

Gray Molina, George (2007). Ethnic Politics in Bolivia: 'Harmony of Inequalities' 1900-2000. CRISE Working Paper, no. 15 [en línea]. Oxford, United Kingdom: Centre for Research on Inequality, Human Security and Ethnicity (CRISE), Queen Elizabeth House (QEH), University of Oxford. Disponible en: https://assets.publishing.service.gov.uk/ media/57a08beeed915d622c000fe3/wp15.pdf (Consultado el 15 de agosto de 2021). 
Gudynas, Eduardo (2015). "El extraño debate sobre la lactosa en la izquierda y en el progresismo. Cambio sustantivo en las discusiones políticas sudamericanas". Diario Página Siete [en línea], 13 de diciembre. Disponible en: https://www.paginasiete.bo/ideas/2015/12/13/ extrano-debate-sobre-lactosa-izquierda-progresismo-79882.html (Consultado el 22 de febrero de 2021).

Gustafson, Bret (2009). "Manipulating Cartographies: Plurinationalism, Autonomy, and Indigenous Resurgence in Bolivia”. Anthropological Quarterly [en línea], 82 (4), pp. 985-1016. Disponible en: https://www.jstor.org/stable/20638677 (Consultado el 15 de agosto de 2021).

Gutiérrez Aguilar, Raquel (2011). "Competing Political Visions and Bolivia's Unfinished Revolution”. Dialectical Anthropology, 35 (3), pp. 275-277.

Huanca, Tomás (comp.) (por publicar). La historia oral de Santos Marka T'ula y del movimiento de caciques apoderados. Bolivia: Taller de Historia Oral Andina. Manuscrito inédito.

Huanca Bustamante, Franz Mauro (2016). "El poder dual en el siglo XXI. La construcción de las vanguardias y el olvido de la revolución”. Iberoamérica social [en línea], 18 de junio. Disponible en: https://iberoamericasocial.com/poder-dual-siglo-xxi/ (Consultado el 15 de agosto de 2021).

Hylton, Forrest (2003). “Tierra común: Caciques, artesanos e intelectuales radicales y la rebelión de Chayanta”, en Hylton, Forrest, et al. Ya es otro tiempo el presente. Cuatro momentos de insurgencia indigena. La Paz, Bolivia: Muela del Diablo Editores, pp. 134-198.

Hylton, Forrest (2004). "El federalismo insurgente: una aproximación a Juan Lero, los comunarios y la Guerra Federal”. Revista Boliviana de Ciencias Sociales y Humanas T’inkazos, Núm. 16, pp. 99-118.

Hylton, Forrest, et al. (2003). Ya es otro tiempo el presente. Cuatro momentos de insurgencia indigena. La Paz, Bolivia: Muela del Diablo Editores.

Irurozqui, Marta (1994). La armonía de las desigualdades. Élites y conflictos de poder en Bolivia, 1880-1920. Madrid, España, Cusco, Perú: Consejo Superior de Investigaciones Científicas, Centro de Estudios Regionales Andinos "Bartolomé de Las Casas".

Laguna, Arian (2019a). Entrevista de Carlos Mesa a René Zavaleta 10 de noviembre de 1983 (1/3) [video]. Disponible en: https://youtu.be/bhvEBX lzn8 (Consultado el 21 de febrero de 2021).

Laguna, Arian (2019b). Entrevista de Carlos Mesa a René Zavaleta 10 de noviembre de 1983 (2/3) [video]. Disponible en: https://youtu.be/AvjEECyxaAg (Consultado el 21 de febrero de 2021).

Laguna, Arian (2019c). Entrevista de Carlos Mesa a René Zavaleta 10 de noviembre de 1983 (3/3) [video]. Disponible en: https://youtu.be/HZXse6lDIKQ (Consultado el 21 de febrero de 2021).

Lalander, Rickard (2017). "Ethnic Rights and the Dilemma of Extractive Development in Plurinational Bolivia”. The International Journal of Human Rights, 21 (4), pp. 464-481.

Mamani Ramírez, Pablo (2017). El estado neocolonial. Una mirada al proceso de la lucha por el poder y sus contradicciones en Bolivia. La Paz, Bolivia: Rincón Ediciones.

EntreDiversidades. Revista de Ciencias Sociales y Humanidades, Vol. 9, Núm. 1 (18), enero-junio 2022. Páginas: $360-381$ ISSN-e: 2007-7610. https://doi.org/10.31644/ED.V9.N1.2022.A15 
Marston, Andrea y Kennemore, Amy (2019). "Extraction, Revolution, Plurinationalism: Rethinking Extractivism from Bolivia”. Latin American Perspectives, 46 (2), pp. 141-160.

Mayorga, Fernando y Rodríguez, Benjamín (2013). "Nacionalismo e indigenismo en el gobierno del MAS”. Temas y debates [en línea], Núm, 20, pp. 97-122. doi: https://doi.org/10.35305/ tyd.v0i20.50 (Consultado el 15 de agosto de 2021).

Mesa Gisbert, Carlos D. (2008). Presidencia sitiada. Memorias de mi gobierno. La Paz, Bolivia: Plural Editores, Fundación Comunidad.

Mesa Gisbert, Carlos D. (2013). La sirena y el charango, ensayo sobre el mestizaje. La Paz, Bolivia: Gisbert editorial, Fundación Comunidad.

Miyachi, Takahiro (2014). Los movimientos étnico-nacionales que interpretan. Análisis comparativo de Bolivia y Ecuador desde la perspectiva constructivista. Tokio, Japón: Fondo Editorial de la Universidad de Tokio. (En japonés, título original: Kaishakusuru Minzokuundou).

Puente, Rafael (2017). “El TIPNIS y la tentación colonial”. Página Siete [en línea], 11 de agosto. Disponible en: https://www.paginasiete.bo/opinion/rafael-puente/2017/8/11/tipnistentacion-colonial-147864.html (Consultado el 10 de mayo de 2021).

Platt, Tristán (2016). Estado boliviano y ayllu andino. Tierra y tributo en el Norte de Potosí. Serie Biblioteca del Bicentenario de Bolivia. La Paz, Bolivia: Vicepresidencia del Estado Plurinacional de Bolivia.

Postero, Nancy (2017). The Indigenous State: Race, Politics, and Performance in Plurinational Bolivia. Oakland, United States of America: University of California Press. Disponible en: https://www.jstor.org/stable/10.1525/j.ctt1pq34b0 (Consultado el 15 de agosto de 2021).

Programa de las Naciones Unidas para el Desarrollo Bolivia (PNUD Bolivia) (2007). Informe Nacional sobre Desarrollo Humano 2007. El estado del Estado en Bolivia [en línea]. La Paz, Bolivia: Oficina de Bolivia del Programa de Naciones Unidas para el Desarrollo. Disponible en: https://www.bo.undp.org/content/bolivia/es/home/library/mdg/informede-desarrollo-humano-2007.html (Consultado el 10 de mayo de 2021).

Reinaga, Fausto (2010). La revolución india, 4a edición. La Paz, Bolivia: Movimiento Indianista Katarista (MINKA).

Rivera Cusicanqui, Silvia (2009). "La contradicción/suplementación entre cultura y desarrollo". Samiri, Año 1, Núm. 3, pp. 76-90.

Rivera Cusicanqui, Silvia (2010). Violencias (re)encubiertas en Bolivia. La Paz, Bolivia: La Mirada Salvaje.

Rivera Cusicanqui, Silvia (2015). Mito y desarrollo en Bolivia. El giro colonial del gobierno del MAS. La Paz, Bolivia: Piedra Rota, Plural Editores.

Rivera Cusicanqui, Silvia (2016). "Estudio introductorio. Estado boliviano y ayllu andino, 30 años después”, en Platt, Tristán (2016). Estado boliviano y ayllu andino. Tierra y tributo en el Norte de Potosí. La Paz, Bolivia: Vicepresidencia del Estado Plurinacional de Bolivia, pp. 15-34.

EntreDiversidades. Revista de Ciencias Sociales y Humanidades, Vol. 9, Núm. 1 (18), enero-junio 2022. Páginas: $360-381$ ISSN-e: 2007-7610. https://doi.org/10.31644/ED.V9.N1.2022.A15 
Rivera Cusicanqui, Silvia y De Sousa Santos, Boaventura (2015). “Conversa del mundo”, en De Sousa Santos, Boaventura (coord.). Revueltas de indignación y otras conversas. La Paz, Bolivia: Proyecto ALICE y Centro de Estudos Sociais (Universidade de Coimbra), pp. 80-123. Disponible en: http://www.boaventuradesousasantos.pt/media/BSS Revueltas(1).pdf (Consultado el 15 de agosto de 2021).

Schavelzon, Salvador (2012). El nacimiento del Estado Plurinacional de Bolivia. Etnografía de una Asamblea Constituyente. La Paz, Bolivia: Grupo de Trabajo Internacional para Asuntos Indígenas (IWGIA), Centro de Estudios Jurídicos e Investigación Social, Plural Editores, Consejo Latinoamericano de Ciencias Sociales.

Shirai, Akira (2007). Lenin inconcluso. Lectura del pensamiento sobre el poder. Tokio, Japón: Kodansha. (En japonés, título original: Mikanno Lenin).

Stern, Steve J. (1988). "Feudalism, Capitalism, and the World-System in the Perspective of Latin America and the Caribbean”. The American Historical Review [en línea], 93 (3), pp. 829-872. doi: https://doi.org/10.2307/1863526 (Consultado el 15 de agosto de 2021).

Svampa, Maristella (2013). “Consenso de los Commodities’ y lenguajes de valoración en América Latina”. Nueva Sociedad [en línea], Núm. 244, pp. 30-46. Disponible en: https://nuso. org/articulo/consenso-de-los-commodities-y-lenguajes-de-valoracion-en-america-latina/ (Consultado el 15 de agosto de 2021).

Tapia, Luis (2002). La producción del conocimiento local. Historia y politica en la obra de René Zavaleta. La Paz, Bolivia: Muela del Diablo editores.

Tapia, Luis (2007). La igualdad es cogobierno. La Paz, Bolivia: Posgrado en Ciencias de Desarrollo, Universidad Mayor San Andrés (CIDES-UMSA), Agencia Sueca para el Desarrollo Internacional (ASDI-SAREC), Plural Editores.

Ticona Alejo, Esteban (2011). "Education and Decolonization in the Work of the Aymara Activist Eduardo Leandro Nina Qhispi”, en Gotkowitz, Laura (ed.). Histories of Race and Racism: The Andes and Mesoamerica from Colonial Times to the Present. Durham, London, United Kingdom: Duke University Press, pp. 240-253.

Thomson, Sinclair (2003). “Cuando solo reinasen los indios': Recuperando la variedad de proyectos anticoloniales entre los comunarios andinos (La Paz, 1740-1781)”, en Hylton, Forrest, et al. Ya es otro tiempo el presente. Cuatro momentos de insurgencia indígena. La Paz, Bolivia: Muela del Diablo Editores, pp. 39-77.

Thomson, Sinclair (2006). Cuando sólo reinasen los indios. La politica aymara en la era de la insurgencia. La Paz, Bolivia: Muela del Diablo Editores, Aruwiyiri.

Umezaki, Kahori (2018). "Bolivia 'fukusū nēshon kokka' no tenbō: Afurokei Boliviajin no jirei kara" [Prospectivas al 'Estado Plurinacional' boliviano: El caso de los afrobolivianos], en Nagano, Yoshiko (ed.) Teikokuto nashonarizumuno gensetsukūkan: kokusaihikaku to sōgorenkei [Espacios discursivos del imperio y del nacionalismo: Comparación e interrelación internacional]. Tokio, Japan: Ochanomizushobō, pp. 241-273.

Valdivia Rivera, Soledad (ed.) (2021). Bolivia at the Crossroads: Politics, Economy, and Environment in a Time of Crisis. London, United Kingdom, New York, United States of America: Routledge.

EntreDiversidades. Revista de Ciencias Sociales y Humanidades, Vol. 9, Núm. 1 (18), enero-junio 2022. Páginas: 360-381 ISSN-e: 2007-7610. https://doi.org/10.31644/ED.V9.N1.2022.A15 
Wanderley, Fernanda (2018). “Extractivismo y traición a los pueblos indígenas del TIPNIS”. Cuestión Agraria [en línea], Vol. 4, pp. 181-201. Disponible en: http://www.ftierra. org/index.php?option $=$ com mtree \&task=att download \&link id $=176 \& \mathrm{cf}$ id $=79$ (Consultado el 15 de agosto de 2021).

Zavaleta Mercado, René (2012 [1973]). "El poder dual en América Latina”. René Zavaleta Mercado. Ensayos 1957-1974. Obra completa Tomo I (reimpresión corregida). La Paz, Bolivia: Plural Editores, pp. 367-513.

Zavaleta Mercado, René (2012 [1962]). “La Revolución Boliviana y el doble poder." René Zavaleta Mercado. Ensayos 1957-1974. Obra completa Tomo I (reimpresión corregida). La Paz, Bolivia: Plural Editores, pp. 535-543.

Zavaleta Mercado, René (2013 [1984]). "Lo nacional-popular en Bolivia (1984)". René Zavaleta Mercado. Ensayos 1975-1984. Obra completa Tomo II. La Paz, Bolivia: Plural Editores, pp. 143-379.

Zavaleta Mercado, René (2013 [1983]). "Las masas en noviembre”. René Zavaleta Mercado. Ensayos 1975-1984. Obra completa Tomo II. La Paz, Bolivia: Plural Editores, pp. 97-142. 